\title{
Original
}

\section{Establishment of a Method for Predicting a Posed Smile from a Straight Face}

\author{
Kei Suzuki $^{1{ }^{1} *}$, Hiroyuki Nakano ${ }^{1) *}$, Tomohiro Yamada $^{2)}$, Sho Mizobuchi ${ }^{1)}$, Kousuke Yasuda $^{2)}$, Safieh Albouga $^{2)}$, Kazuya Inoue $^{1)}$, \\ Mayumi Matsumura $^{1)}$, Shiho Tajiri ${ }^{2)}$, Katsuaki Mishima ${ }^{3)}$, Yoshihide Mori' ${ }^{2)}$ and Takaki Ueno ${ }^{1)}$ \\ ${ }^{1)}$ Department of Oral Surgery, Osaka Medical College, Osaka, Japan \\ 2) Division of Oral and Maxillofacial Surgery, Department of Maxillofacial \\ Diagnostic and Surgical Sciences, Faculty of Dental Science, Kyushu University, Fukuoka, Japan \\ 3) Department of Oral and Maxillofacial Surgery, Graduate School of Medicine, Yamaguchi University, Yamaguchi, Japan \\ (Accepted for publication, April 23, 2021)
}

\begin{abstract}
Good facial expression is an important goal of orthognathic surgery because facial expression has a considerably greater influence on humans' aesthetic judgements than facial profile alone. However, to date, no reports have attempted to predict post-operative smiles from straight faces. The aim of this study was to evaluate the effectiveness of different techniques to create a posed smile (virtual) from a straight face (original). Twenty-five volunteers with no medical history that would interfere with a straight face or a posed smile were enrolled. After creating homologous models from the straight face and posed smile models, we assessed the ability of the principal component (PC) method and the improved Manchester (i$\mathrm{M})$ method to create a posed smile (virtual) from a straight face (original). Positive errors for the PC and i-M were $1.4 \pm 0.5$ $\mathrm{mm}, 0.9 \pm 0.4 \mathrm{~mm}$, respectively, and there was a significant difference. Although there were significant differences in error, the error of two methods, including homologous modeling techniques and principal component analysis, were clinically small and useful for predicting change in facial expression.
\end{abstract}

Key words: Homologous model, Posed smile, Predicting the face, Principal component analysis, Straight face

\section{Introduction}

Orthognatic surgery represents a major portion of oral and maxillofacial surgeries. Good facial expression is an important goal of orthognathic surgery because facial expression has a considerably greater influence on humans' aesthetic judgements than facial profile alone. Therefore, many studies on pre- and post-operative facial changes and how to predict them have been published ${ }^{1,2)}$. In our previous study, gender differences in posed smiles and the characteristics of posed smiles by female patients with Class 3 malocclusion before and after orthognathic surgery were investigated using principal component analysis $(\mathrm{PCA})^{3)}$. However, to date, no reports have attempted to predict post-operative smiles.

The homologous modeling technique has recently gained prominence as a method for creating a database of the face and jaw bones ${ }^{4-7}$. This technique can express an object shape as a polygon of the same topological structure with the same anatomical landmarks. To enhance the anatomical accuracy, facial reconstruction can be performed by adding a facial muscle model to the bone model. In the homologous model technique, the XYZ coordinates of the vertices can be calculated back from an arbitrary principal component (PC) value to create an average model $^{8-10)}$. Therefore, a regression equation that can predict the $\mathrm{PC}$ values of a "posed smile (original)" using the PC values of a "straight face (original)" of the same patient can also be used to create a "posed smile (virtual)" from the straight face (original).

* These authors contributed equally to this work.

Correspondence to: Dr. Hiroyuki Nakano, Department of Oral Surgery, Osaka Medical College, 2-7 Daigaku-machi, Takatsuki City, Osaka 569-8686, Japan; Phone: +8172-683-1221; E-mail: ora099@osaka-med.ac.jp
In this study, we created a posed smile (virtual) from a straight face (original) using two different techniques and evaluated the accuracy of prediction for each technique.

\section{Patients \\ Materials and Methods}

Twenty-five volunteers with no medical history associated with a straight face or a posed smile were enrolled in the study. The experimental protocols were approved by the Ethics Committee of the Faculty of Dental Science, Kyushu University, Fukuoka, Japan (30-295) and were performed in line with the principles of the Declaration of Helsinki. All participants gave their informed consent to participate in the study.

\section{Imaging and analysis}

Facial image construction and homologous modeling were based on a previous report ${ }^{5}$. During imaging, the subjects were seated and the head was positioned without any head fixation. HBM-Rugle (Medic Engineering; Kyoto, Japan) image measurement software in stereolithographic format and Homologous Body Modeling software (Digital Human Technology; Tokyo, Japan) were used to construct a 3D image of the face for each subject and plot 11 landmarks on the surface of the 3D model $^{5}$. Template models of the face consisting of approximately 6887 polygons were generated using Geomagic Studio 9 (Geomagic; NC, USA). The template model was automatically fitted to the individually scanned point cloud of the face by minimizing external and internal energy functions. The external energy function was based on the Euclidian distance between data points of the template model and those of the pa- 
J.Hard Tissue Biology Vol. 30(3): 221-224, 2021

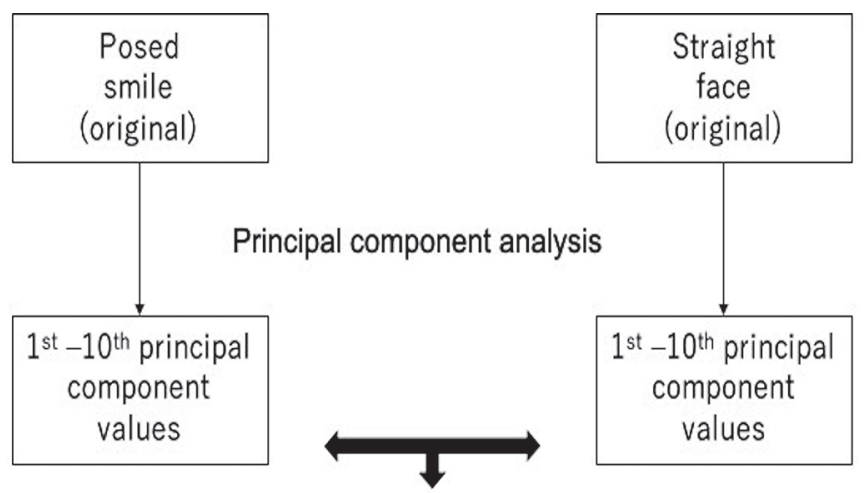

Regression equation

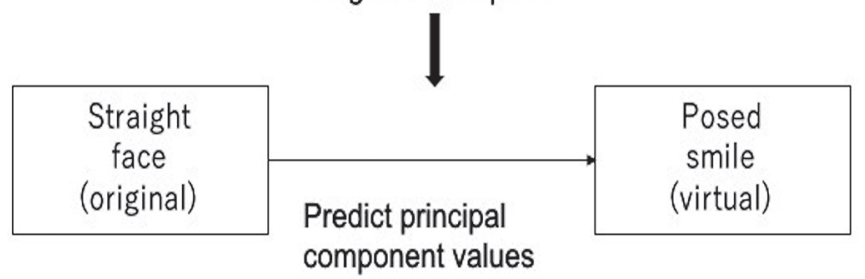

Figure 1. Algorithm of the principal component method. We created a regression equation to predict the PC value of the original posed smile model from the first $10 \mathrm{PC}$ values of the original straight face model. A virtual posed smile model was then created from the PC values predicted by the regression equation.

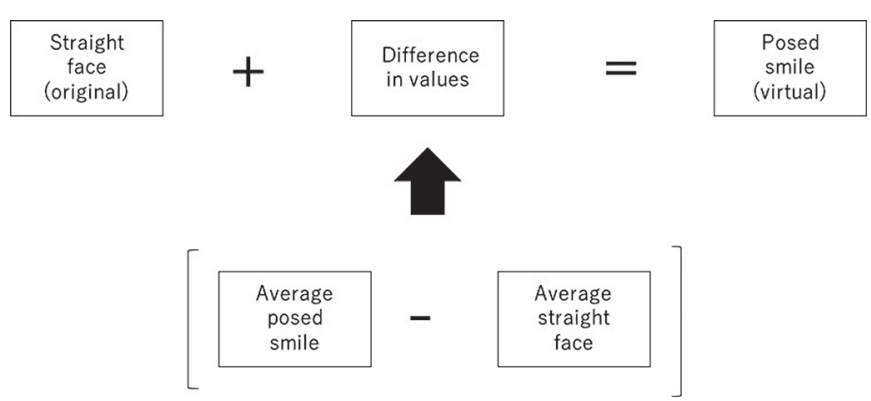

Figure 2. Algorithm of the improved Manchester (i-M) method. First, differences between the straight face and the posed smile were identified in the XYZ coordinates of the vertices of the polygon. Then, the differences were added to the XYZ coordinates of the vertices of the polygon of the straight face.

tient's database. The internal energy function was based on local deformation of the template model. The vertices of the template model were considered as anatomical landmarks that were fitted to landmarks, whereas the vertices generated from the subdivision surface were fitted to the measured point clouds with minimum deformation of the initial template model. The prediction of a posed smile from a straight face was then performed using each of three methods, as follows.

-Principal component (PC) method (Fig. 1)

Using PC analysis of the 48 faces (24 original straight faces and 24 original posed smiles), we created a regression equation to predict the $\mathrm{PC}$ value of the original posed smile model from the first $10 \mathrm{PC}$ values of the original straight face model. A virtual posed smile model was then created from the PC values predicted by the regression equation, and this was compared with the original posed smile model. This procedure was repeated 25 times. The original and virtual posed smiles were com-
Table 1. The results of principal component analysisof the straight face

\begin{tabular}{cccc}
\hline $\begin{array}{c}\text { Principal } \\
\text { component }\end{array}$ & $\begin{array}{c}\text { Principal compo- } \\
\text { nent value }\end{array}$ & $\begin{array}{c}\text { Contribution ratio } \\
(\%)\end{array}$ & $\begin{array}{c}\text { Cumulative contribution } \\
\text { ratio (\%) }\end{array}$ \\
\hline 1 & 6932.5 & 32.8 & 32.8 \\
2 & 4046.2 & 19.1 & 51.9 \\
3 & 2210.8 & 10.4 & 62.3 \\
4 & 1435.0 & 6.8 & 69.1 \\
5 & 1214.4 & 5.7 & 74.8 \\
6 & 1036.3 & 4.9 & 79.7 \\
7 & 704.8 & 3.3 & 83.0 \\
8 & 534.3 & 2.5 & 85.5 \\
9 & 491.4 & 2.3 & 87.8 \\
10 & 434.4 & 2.1 & 89.9 \\
11 & 339.8 & 1.6 & 91.5 \\
12 & 313.2 & 1.5 & 93.0 \\
13 & 226.6 & 1.1 & 94.1
\end{tabular}

Table 2. The results of principal component analysis of posed smile

\begin{tabular}{cccc}
\hline $\begin{array}{c}\text { Principal } \\
\text { component }\end{array}$ & $\begin{array}{c}\text { Principal compo- } \\
\text { nent value }\end{array}$ & $\begin{array}{c}\text { Contribution ratio } \\
(\%)\end{array}$ & $\begin{array}{c}\text { Cumulative contribution } \\
\text { ratio (\%) }\end{array}$ \\
\hline 1 & 7272.8 & 34.4 & 34.4 \\
2 & 3471.6 & 16.4 & 50.8 \\
3 & 2160.5 & 10.2 & 61.0 \\
4 & 1480.2 & 7.0 & 68.0 \\
5 & 1320.9 & 6.2 & 74.2 \\
6 & 1046.0 & 4.9 & 79.1 \\
7 & 743.4 & 3.5 & 82.6 \\
8 & 557.0 & 2.6 & 85.2 \\
9 & 480.0 & 2.3 & 87.5 \\
10 & 402.1 & 1.9 & 89.4 \\
11 & 353.6 & 1.7 & 91.1 \\
12 & 318.4 & 1.5 & 92.6 \\
13 & 264.1 & 1.2 & 93.8 \\
14 & 218.3 & 1.0 & 94.8 \\
\hline
\end{tabular}

pared, and the error between them was measured.

-Improved Manchester method (i-M method) (Fig. 2)

In the Manchester method, facial reconstruction is performed by adding an average thickness of soft tissue such as muscle, fat, and skin onto the skull using anatomical landmarks ${ }^{11)}$. In the present study, we aimed to improve this technique and apply it to the prediction of a posed smile (virtual) from a straight face (original). First, differences between the straight face and the posed smile were identified in the XYZ coordinates of the vertices of the polygon. The differences were added to the $\mathrm{XYZ}$ coordinates of the vertices of the polygon of the straight face, and this procedure was repeated 25 times. The original and virtual posed smiles were compared, and the error between them was measured.

\section{Statistical analysis}

The faces were analyzed using PCA and multiple regression analysis. All descriptive data analyses were performed with JMP 5.1.2 (SAS Institute Inc; Cary, NC, USA).

\section{Results}

Among the straight face image sets, the contribution of the most important PC was $32.8 \%$, and 10 PCs explained $>90 \%$ of the total variance (Table 1).

Among the posed smile image sets, the contribution of the most important PC was $34.3 \%$, and 11 PCs explained $>90 \%$ of the total variance (Table 2).

Positive errors in the PC and i-M were $1.4 \pm 0.5 \mathrm{~mm}$ and $0.9 \pm 0.4$ $\mathrm{mm}$, respectively (Fig. 3). 
Kei Suzuki et al:: Establishment of a Method for Predicting a Face

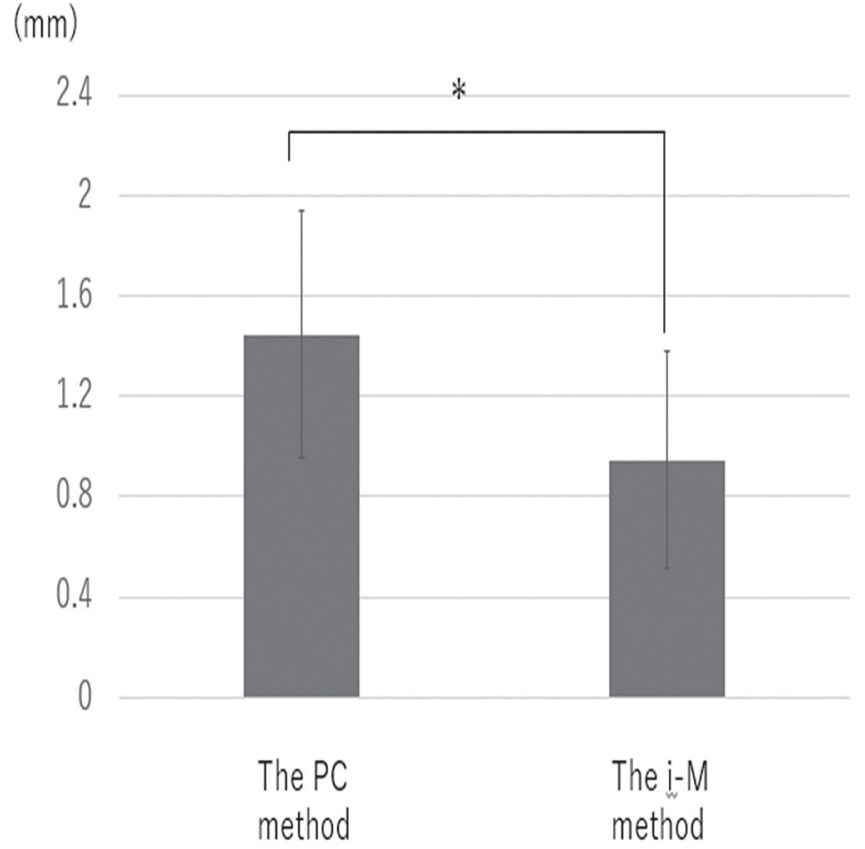

Figure 3. Positive errors between the original and virtual posed smiles for the three methods. Positive errors in the PC and i-M were $1.4 \pm 0.5 \mathrm{~mm}$ and $0.9 \pm 0.4 \mathrm{~mm}$, respectively.

\section{Discussion}

Traditional facial reconstruction methods are based on manual procedures and produce 2D portraits or 3D sculptures. There are three common steps in these methods: (1) the raw skull (or a replica) is equipped with a sparse set of anatomical landmarks, (2) an average soft tissue thickness is applied to each skull landmark to estimate a corresponding landmark on the face, and (3) a face fitting the estimated landmarks is drawn or sculpted. Most practitioners add a facial muscle model to enrich the anatomical accuracy of the reconstruction, termed the Manchester method.

Computer animation software packages are a more recent development that use the same methodology as manual methods, and allow the user to tune some of the modeling parameters and combine human expertise with the flexibility of the software ${ }^{12)}$.

Morphological measurement of the human body can be obtained using anatomical landmarks and their distances and angles; however, evaluation by this method is localized, and it is difficult to evaluate the overall characteristics. Homologous modeling technique is increasingly being used as morphological measurement method in which scanned surface shape data are represented using the same number of data points as in topology defined based on the anatomy ${ }^{13)}$. Recent reports have indicated the efficacy of homologous modeling for 3D morphological analysis of human teeth and faces, which are composed of relatively smooth surfaces. In addition, analysis of homologous models using multivariate statistical methods enables the extraction of shape variations that are impossible to understand using linear measurements. We have previously evaluated the difference between "straight face" and "posed smile" in healthy subjects using homologous modeling technique ( $^{5}$ and that between mandibular condyles on the deviated and non-deviated sides in patients with jaw deviation ${ }^{6}$. The homologous modeling technique can calculate the averages of PC values obtained by PC analysis, and the $\mathrm{XYZ}$ coordinates can be calculated from the average values to visualize the virtual average shape.

In the present study, a homologous model with the same shape as the scanned model was created to apply morphing methodology and fabricate a 3D facial model. Because the homologous model in this study was created from the same original template, the number of vertices is the same and they show one-to-one correspondence, representing the morphology of the scanned model so that morphing methodology can be applied.

We considered that the main component values of a posed smile could be predicted from the main component values of a straight face using multiple regression analysis. Using the PC method, the error between the original posed smile and the virtual posed smile was $1.4 \pm 0.5$ $\mathrm{mm}$, which was higher than that using the i-M method but can still be considered a good result clinically.

As the i-M method uses data from an average image, it may not be suitable for patients with jaw deformities. In contrast, the PC method can be used in various situations. Although there were significant differences in error, the error of PC method and i-M method, including homologous modeling techniques and principal component analysis, were clinically small and useful for predicting change in facial expression.

In the future, I think it will be necessary to use and evaluate the two methods in various situations.

\section{Acknowledgements}

The authors wish to thank Ms. Kaori Tanijiri and Mr. Toyohisa Tanijiri at Medic Engineering Co. Ltd. for their technical support in software implementation.

This work was supported by a grant from JSPS KAKENHI (No. 20K18708).

\section{Conflict of Interest}

The authors have declared that they have no conflicts of interest.

\section{References}

1. Jung YJ, Kim MJ and Baek SH. Hard and soft tissue changes after correction of mandibular prognathism and facial asymmetry by mandibular setback surgery: Three-dimensional analysis using computerized tomography. Oral Surg Oral Med Oral Pathol Oral Radiol Endod 107: 763-771, 2009

2. Almeida RC, Cevidanes LHS, Carvalho FAR, Motta AT and Almeida MAO. Soft tissue response to mandibular advancement using 3D CBCT scanning. Int J Oral Maxillofac Surg 40: 353-359, 2011

3. Mishima K, Shiraishi M, Kawai Y, Umeda H, Nakano H and Ueyama Y. Characteristics of posed smiles for class 3 female patients before and after osteotomy using principal component analysis. J Craniofac Surg 27: 1754-1758, 2016

4. Nakano H, Mizobuchi S, Suzuki K, Inoue K, Yamamoto N, Omori M, Kato-Kogoe N, Nakajima Y, Kimura Y, Mishima K and Ueno T. Evaluation of the utility of homologous modeling and principal component analysis for sex determination of the mandible. J Hard Tissue Biol 30: 69-71, 2021

5. Yasuda K, Nakano H, Yamada T, Albougha S, Inoue K, Nakashima A, Kamata YU, Sugiyama G, Tajiri S, Sumida T, Mishima K and Mori Y. Identifying differences between a straight face and posed smile using the homologous modeling technique and the principal component analysis. J Craniofac Surg 30: 2378-2380, 2019

6. Inoue K, Nakano H, Sumida T, Yamada T, Otawa N, Fukuda N, Nakajima Y, Kumamaru W, Mishima K, Kouchi M, Takahashi I and Mori Y. A novel measurement method for the morphology of the 
mandibular ramus using homologous modeling. Dentomaxillofac Radiol 44: 20150062, 2015

7. Suzuki K, Nakano H, Inoue K, Nakajima Y, Mizobuchi S, Omori M, Kato-Kogoe N, Mishima K and Ueno T. Examination of new parameters for sex determination of mandible using Japanese computer tomography data. Dentomaxillofac Radiol 49: 20190282, 2020

8. Biwasaka H, Aoki Y, Takahashi Y, Takahashi Y, Fukuta M, Usui A, Hosokai Y, Saito H, Funayama M, Fujita S, Takamiya M and Dewa $\mathrm{K}$. A quantitative morphological analysis of three-dimensional CT coxal bone images of contemporary Japanese using homologous models for sex and age estimation. Leg Med (Tokyo) 36: 1-8, 2019

9. Matsuoka A, Yoshioka F, Ozawa S and Takebe J. Development of three-dimensional facial expression models using morphing methods for fabricating facial prostheses. J Prosthodont Res 63: 66-72,
2019

10. Imaizumi K, Taniguchi K, Ogawa Y, Matsuzaki K, Negata T, Mochimaru M and Kouchi M. Three-dimensional analyses of aging-induced alterations in facial shape: a longitudinal study of 171 Japanese males. Int Legal Med 129: 385-393, 2015

11. Wilkinson C. Computerized forensic facial reconstruction: a review of current systems. Forensic Sci Med Pathol 1: 173-177, 2005

12. Miyasaka S, Yoshinoa M, Imaizumi K and Seta S. The computer-aided facial reconstruction system. Forensic Sci Int 74: 155-165, 1995

13. Kouchi M and Mochimaru M. Simulation of the body shape after weight change for health-care services. In: Advances in Applied Digital Human Modeling, ed by Duffy V. G, CRC Press, Indiana, 217-222, 2010 